DOI: 10.20472/EFC.2021.015.012

\title{
OSARUMWENSE OSABUOHIEN-IRABOR
}

Department of International Economics, School of Economics, Ural Federal University, Russian Federation

\section{IGOR MIKHAILOVICH DRAPKIN}

Department of International Economics, School of Economics, Ural Federal University, Russian Federation

\section{OUTWARD FDI AND INTERNATIONAL TRADE: THE STUDY OF CAUSAL EFFECTS}

\begin{abstract}
:
Numerous studies have examined whether the interrelationship between outward foreign direct investment (OFDI) and international trade are complementary or substitutive. However, one major concern of policymakers is the possibility of OFDI precipitating de-industrialization and jobs losses of domestic economy. This study critically addresses these views by examining the interaction between OFDI and disaggregate international trade based on world bank country income classification which includes, the low income, lower-middle income, upper-middle income, and High income for a panel of 179 countries for the period of 2003 - 2019. Based on dynamic panel data model for system-GMM, empirical findings show that OFDI has negative and significant effects on exports and imports of low-income countries, an indication of a substitutional relationship. Regarding the effects of exports on OFDI, and with exception of low-income countries, we found a positive and significant relationship for in all income cluster, an indication of a complementarity relationship. This shows that home country's export is an important facilitator of OFDI. Overall, our empirical results support complementary effects on the dynamic interplay between OFDI and disaggregate international trade, suggesting a greater competitiveness in foreign markets as well as an increase in commercial integration.
\end{abstract}

\section{Keywords:}

Outward FDI, Trade, Export, Import, Income economies, Dynamic panel data

JEL Classification: F10, F21, F43 


\section{Introduction}

Foreign direct investment (FDI) and international trade are two major components in international economic relations. Over the past decade, the flow volumes of these economic factors have increased due to waves of globalization and liberalization of trade and investment. The numbers of parent multinational corporation (hereafter referred to MNCs) also increased from 7000 in 1970 to 82000 in 2008, and its global gross output of foreign affiliate as at 2014 grew to US $\$ 20$ trillion dollars from US $\$ 7$ trillion dollars in 2000 (Alguacil, Cuadros \& Orts, 2008). Whilst global IFDI grew from US $\$ 7.5$ trillion to US $\$ 19$ trillion between 2000 to 2010 , OFDI increased by 16 per cent from US $\$ 1,429$ billion as at 2010, to reach an estimated US\$1.66 trillion in 2011 (UNCTAD, 2012). However, these flows fell by 49 per cent in 2020 compares to 2019 due to economic crisis caused by COVID-19 global pandemic (UNCTAD, 2021). These growth and contractions of FDI flows has attracted significant attention from researchers, international investors, and policy makers. And this has led to different strands of literatures, and of particular interest is on studies which aim to examine whether OFDI flows substitute or complements home country trade.

Whilst numerous empirical studies that have endorsed the substitutional theory of OFDI and trade relationship (Zhao, Liu, Wei, \& Andreosso-O'Callaghan, 2017; Bhasin \& Kapoor, 2020), other empirical findings showed that OFDI impact on trade are significant and positive indicating a complementarity relationship (Zhi-yuan, 2017; Zhou, 2020; Albulescu \& Goyeau, 2019). Albulescu \& Goyeau (2019) empirical studies indicate that OFDI impacts on trade in Central and Eastern European (CEE) countries are complementary. Due to the specificity of countries, results for FDI and trade interrelationship have also been detected as mixed (Dauti, 2016). Nevertheless, there is the need to examine this relation based on world bank income classification such as low, lowmiddle, upper-middle, and high-income economies, in order to ascertain which of the economies cluster are consistent with complementarity or substitutive effects assertion. Apart from studies such as Joshua, Rotimi, \& Sarkodie (2020) which examined IFDI flow and income groups, very little attention has been paid to examining the effects of OFDI on country's trade with regards to country's income economies.

\section{Methodology and data}

We use panel data of 179 (economies) countries and classify them using world bank income-level classification for the period 2003 - 2019. All data are obtained from both UNCTAD and World Development Indicators (WDI). Data includes, OFDI, IFDI, exports (EXP) \& import (IMP) of goods and services, Gross Domestic product (GDP), Time spend dealing with export requirements as part of government regulations (TEXP), Trade tariff (TRDT), Human development indicator (HDI), Quality of infrastructure (QINF) and state debts (DEBT). This study explores the pattern of causal effects between OFDI and international trade across World Bank country income clusters such as the low income, lower-middle income, upper-middle income, and High income. To overcome problems associated with unobserved heterogeneity, potential endogeneity and omitted variable bias in the dynamic panel model, the system GMM techniques by Blundell \& Bond, (1998) is employed. The model specifications include,

Model I

$$
X_{i, t}=\beta_{i}+\beta_{1} X_{(i, t-1)}+\beta_{2} Y_{(i, t)}+\beta_{3} Z_{(i, t)}+u_{i}+\varepsilon_{i t}
$$


Model II

$$
Y_{i, t}=\beta_{i}+\beta_{1} Y_{(i, t-1)}+\beta_{2} X_{(i, t)}+\beta_{3} Z_{(i, t)}+u_{i}+\varepsilon_{i t}
$$

Where,

In model I, $X$ is the dependent variable indicated by the disaggregate trade variable of log of $E X P$ and $\log$ of $I M P, Y$ is explanatory variable of interest indicated by the log of OFDI. For model II, $Y$ is the $\log$ of $O F D I$ as dependent variable, $X$ is explanatory variable of interest indicated by the $\log$ of $E X P$ and $\log$ of $I M P, Z$ is the set of other explanatory variables such as $\log I F D I, \log G D P, \log$ $T E X P, H D I, \log T R D T, \log Q I N F, \log D E B T, \varepsilon_{i, t}$ indicates the error term, $(i, t)$ indicates country ${ }^{\prime} i$ ' in year ' $t$ ', $\beta_{i}$ and $u_{i}$ are country and time specific-effects respectively.

\section{Empirical results}

Table 1 reports the effects of OFDI flow on country's export, and Table 2 shows the results for the reversed effects of OFDI on export (i.e. export supporting FDI) for countries across different income classifications such as the low income, lower-middle income, upper-middle income, High income, the middle and world (all-income) income. A pre-analysis tests conducted, which includes statistical properties of data, multicollinearity, panel data unit root and endogeneity tests, shows reveals absence of multicollinearity in the regression model. The Wu Hausman test reports absence of endogeneity problem in both models and based on the null hypothesis of common unit roots are rejected for all variables across sample data. However, for the sake of brevity, the pre analysis tests are not displayed in this paper but will be available on request. We construct two dynamic models (Model I \& II) shown in equation (1) and (2) to capture the interrelationship between disaggregate trade and OFDI.

Table 1 results shows that the effects of OFDI on export and countries with low-middle, uppermiddle, high, middle and world (all-come) income are positive and highly statistically significant, except for low income. This is consistent with some previous studies such as Albulescu \& Goyeau (2019) which supports a complementarity effect of OFDI flow on exports. Although the estimated coefficients of OFDI effects on exports across income clusters varies, but the impacts are positive and significant for almost all income groups (Table 1). However, there is a negative effect of OFDI on exports for low income economies, suggesting a substitutional relationship. The impact of inward FDI home exports are also positive and significant for all income economies. This finding is consistent with earlier studies which suggests that inward FDI is exports-oriented and provides a complementarity effect (Sharmiladevi, 2020). The lagged exports variable for all income groups examined are positive and significant. This indicates a demonstration effects confirming high persistence of the export variable. Quality of infrastructure (QINF) for upper-middle, and high income also positively significant. This suggest that a per cent increase in QINF for such economies will boost exports by $11.8 \%, 71.8 \%$ respectively, see Table 1 . This finding partly confirmed previous empirical results that infrastructure positively promotes exports (Lorz, 2020; Ismail, 2021). Table 2 estimation results indicate that $5.8 \%, 16.9 \%$ and $22.9 \%$ impacts of export-supporting FDI exist for low-middle, upper middle, and high-income countries based on world bank income clusters. This result is consistent with Krautheim (2013) paper which argues that country's trade provides support for MNCs activities, particularly its outbound FDI. 
Previous OFDI flow shows a positive and significant effects on the current FDI for all income economies classification. This suggests that past FDI is a good predictor of current FDI (Mazouz, Wood, Yin, \& Zhang, 2021; Aziz, 2018). The OFDI lag of one-year period in the explanatory model are used to assess its dynamic effects. Tables 1 and 2 estimations reports AR (1) to be significant, but the model diagnostics results of Arellano-Bond tests AR (2) statistics for serial correlation are insignificant, suggesting the absence of second-order autocorrelation in the residuals for all income economies specifications. Hansen tests of over identifying restrictions are also insignificant indicating that the instruments are valid and not correlated with the residual.

Table 1: Two-step system GMM estimation results for effects of outward FDI on EXP

\begin{tabular}{|c|c|c|c|c|c|}
\hline & \multicolumn{4}{|c|}{ World Bank Income Classification } & \multirow[b]{2}{*}{ All Income } \\
\hline & Low & Low-Middle & Upper-Middle & High & \\
\hline Variables & (1) & $(2)$ & (3) & (4) & (5) \\
\hline Lagged EXP & $\begin{array}{l}0.746 * * * \\
(34.450)\end{array}$ & $\begin{array}{l}0.741 * * * \\
(180.080)\end{array}$ & $\begin{array}{l}0.561 * * * \\
(33.750)\end{array}$ & $\begin{array}{l}0.674 * * * \\
(94.230)\end{array}$ & $\begin{array}{l}0.237 * * * \\
(8.350)\end{array}$ \\
\hline IFDI & $\begin{array}{l}0.284 * * * \\
(10.370)\end{array}$ & $\begin{array}{l}-0.031 * * * \\
(-2.790)\end{array}$ & $\begin{array}{l}0.315 * * * \\
(6.780)\end{array}$ & $\begin{array}{l}0.054 * * * \\
(8.730)\end{array}$ & $\begin{array}{l}0.110 * * * \\
(4.860)\end{array}$ \\
\hline OFDI & $\begin{array}{l}-0.174 * * * \\
(-4.060)\end{array}$ & $\begin{array}{l}0.025 * * * \\
(7.820)\end{array}$ & $\begin{array}{l}0.214 * * * \\
(9.090)\end{array}$ & $\begin{array}{l}0.018 * * * \\
(4.000)\end{array}$ & $\begin{array}{l}0.023 * * \\
(2.510) \\
\end{array}$ \\
\hline GDP & $\begin{array}{l}0.287 * * * \\
(4.480)\end{array}$ & $\begin{array}{l}-0.180 * * \\
(-2.090)\end{array}$ & $\begin{array}{l}0.450 * * * \\
(3.360)\end{array}$ & $\begin{array}{l}0.099 * * * \\
(4.500)\end{array}$ & $\begin{array}{l}0.121 * * \\
(2.430) \\
\end{array}$ \\
\hline TEXP & $\begin{array}{l}-0.691 \\
(-0.910) \\
\end{array}$ & $\begin{array}{l}-0.864 * * * \\
(-4.230)\end{array}$ & $\begin{array}{l}0.540 * * * \\
(5.990)\end{array}$ & $\begin{array}{l}-0.491 * * * \\
(-9.340)\end{array}$ & $\begin{array}{l}-0.621^{*} \\
(-1.700) \\
\end{array}$ \\
\hline HDI & $\begin{array}{l}-1.007 * \\
(-1.800) \\
\end{array}$ & $\begin{array}{l}-0.242 \\
(-1.110) \\
\end{array}$ & $\begin{array}{l}0.462 * * * \\
(2.720)\end{array}$ & $\begin{array}{l}-0.676 * * * \\
(-4.670)\end{array}$ & $\begin{array}{l}0.395 * * * \\
(3.190)\end{array}$ \\
\hline TRDT & $\begin{array}{l}0.161 \\
(1.130)\end{array}$ & $\begin{array}{l}0.255 * * * \\
(7.170)\end{array}$ & $\begin{array}{l}-0.101^{*} \\
(-1.760)\end{array}$ & $\begin{array}{l}0.127 * * \\
(2.522) \\
\end{array}$ & $\begin{array}{l}0.179 * * * \\
(4.190)\end{array}$ \\
\hline QINF & $\begin{array}{l}-0.181 \\
(-0.830) \\
\end{array}$ & $\begin{array}{l}-0.760 * * * \\
(-10.800)\end{array}$ & $\begin{array}{l}0.118 * * * \\
(5.480)\end{array}$ & $\begin{array}{l}0.718 * * * \\
(15.790)\end{array}$ & $\begin{array}{l}0.513 * * * \\
(3.450)\end{array}$ \\
\hline DEBT & $\begin{array}{l}-0.234^{*} \\
(-1.810) \\
\end{array}$ & $\begin{array}{l}0.354 * * * \\
(7.270)\end{array}$ & $\begin{array}{l}0.249 * * * \\
(5.060)\end{array}$ & $\begin{array}{l}-0.757 * * * \\
(-24.230) \\
\end{array}$ & $\begin{array}{l}-0.151 * * \\
(-2.190) \\
\end{array}$ \\
\hline Constant & $\begin{array}{l}2.045 \\
(1.400)\end{array}$ & $\begin{array}{l}1.566 * * \\
(10.860) \\
\end{array}$ & $\begin{array}{l}-2.749 * * * \\
(-6.480)\end{array}$ & $\begin{array}{l}1.948 * * * \\
(10.870)\end{array}$ & $\begin{array}{l}5.134 * * * \\
(6.210)\end{array}$ \\
\hline Nos. of Obs/Grand & $400 / 4260$ & $704 / 7480$ & $880 / 9350$ & $880 / 9350$ & $2864 / 30430$ \\
\hline Nos. of Instrument & 22 & 43 & 50 & 52 & 88 \\
\hline Nos. of Groups & 25 & 44 & 56 & 55 & 179 \\
\hline Wald test p-value & 0.000 & 0.000 & 0.000 & 0.000 & 0.000 \\
\hline AR (1) $p$-value & 0.006 & 0.005 & 0.012 & 0.034 & 0.000 \\
\hline AR (2) $p$-value & 0.273 & 0.307 & 0.211 & 0.604 & 0.304 \\
\hline Hansen $p$-value & 0.687 & 0.375 & 0.210 & 0.242 & 0.291 \\
\hline
\end{tabular}

Source: Author's calculations

$\mathrm{t}$-statistics are in parentheses and all standard errors are two-step, significance: $* \mathrm{p}<0.1 ; * * \mathrm{p}<0.05 ; * * * \mathrm{p}<0.01$ 
Table 2: Two-step system GMM estimation results for exports supporting outward FDI

\begin{tabular}{|c|c|c|c|c|c|}
\hline & \multicolumn{4}{|c|}{ World Bank Income Classification } & \\
\hline & Low & Low-Middle & Upper-Middle & High & All Income \\
\hline Variables & (1) & $(2)$ & (3) & (4) & (5) \\
\hline Lagged OFDI & $\begin{array}{l}0.217 * * * \\
(4.120)\end{array}$ & $\begin{array}{l}0.171 * * * \\
(6.660)\end{array}$ & $\begin{array}{l}0.091 * * * \\
(4.890)\end{array}$ & $\begin{array}{l}0.108 * * * \\
(5.170)\end{array}$ & $\begin{array}{l}0.227 * * * \\
(11.480)\end{array}$ \\
\hline IFDI & $\begin{array}{l}0.272 * \\
(1.740)\end{array}$ & $\begin{array}{l}-0.271 * * * \\
(-4.410)\end{array}$ & $\begin{array}{l}0.765^{* * * *} \\
(14.630)\end{array}$ & $\begin{array}{l}1.004 * * * \\
(14.340)\end{array}$ & $\begin{array}{l}0.330 * * * \\
(7.910)\end{array}$ \\
\hline EXP & $\begin{array}{l}-0.199 * * * \\
(-4.010)\end{array}$ & $\begin{array}{l}0.058 * * \\
(2.590)\end{array}$ & $\begin{array}{l}0.169 * * * \\
(11.450)\end{array}$ & $\begin{array}{l}0.229 * * * \\
(-3.430)\end{array}$ & $\begin{array}{l}0.471 * * * \\
(2.780)\end{array}$ \\
\hline GDP & $\begin{array}{l}0.327 * * * \\
(3.170)\end{array}$ & $\begin{array}{l}0.197 * * * \\
(3.400)\end{array}$ & $\begin{array}{l}-0.367 * * * \\
(-2.690)\end{array}$ & $\begin{array}{l}0.731 * * * \\
(10.600)\end{array}$ & $\begin{array}{l}0.772 * * * \\
(0.001)\end{array}$ \\
\hline TEXP & $\begin{array}{l}0.692 \\
(0.610)\end{array}$ & $\begin{array}{l}0.513 * * * \\
(2.620)\end{array}$ & $\begin{array}{l}-0.811 * * \\
(-2.050)\end{array}$ & $\begin{array}{l}-0.513 * * * \\
(-10.440)\end{array}$ & $\begin{array}{l}-0.312 * * * \\
(-4.970)\end{array}$ \\
\hline HDI & $\begin{array}{l}0.408^{*} \\
(1.740)\end{array}$ & $\begin{array}{l}1.005^{* * *} \\
(4.600)\end{array}$ & $\begin{array}{l}0.684 * * * \\
(5.480)\end{array}$ & $\begin{array}{l}-0.122 * * * \\
(-4.590)\end{array}$ & $\begin{array}{l}0.310 * * * \\
(3.970)\end{array}$ \\
\hline TRDT & $\begin{array}{l}0.455 * * \\
(2.560) \\
\end{array}$ & $\begin{array}{l}0.353 * * * \\
(7.830)\end{array}$ & $\begin{array}{l}-0.280 * \\
(-1.740) \\
\end{array}$ & $\begin{array}{l}0.298 * * * \\
(2.760)\end{array}$ & $\begin{array}{l}-0.014 \\
(-0.250) \\
\end{array}$ \\
\hline QINF & $\begin{array}{l}-0.075 \\
(-0.070)\end{array}$ & $\begin{array}{l}0.819 * * \\
(2.690)\end{array}$ & $\begin{array}{l}-0.605^{*} \\
(-1.980)\end{array}$ & $\begin{array}{l}0.554 * * * \\
(5.000)\end{array}$ & $\begin{array}{l}0.501 * * * \\
(3.220)\end{array}$ \\
\hline DEBT & $\begin{array}{l}-0.522 * * * \\
(-3.380)\end{array}$ & $\begin{array}{l}0.456 * * \\
(2.200)\end{array}$ & $\begin{array}{l}-0.878^{* * * *} \\
(-4.930)\end{array}$ & $\begin{array}{l}-0.876^{* * * *} \\
(-3.380)\end{array}$ & $\begin{array}{l}-0.353^{* * * *} \\
(-2.91)\end{array}$ \\
\hline Constant & $\begin{array}{l}-3.516 \\
(11.260)\end{array}$ & $\begin{array}{l}-0.807 * * \\
(-2.140)\end{array}$ & $\begin{array}{l}-0.487 \\
(-0.310) \\
\end{array}$ & $\begin{array}{l}3.258 * * * \\
(7.690)\end{array}$ & $\begin{array}{l}1.864^{*} \\
(1.890)\end{array}$ \\
\hline Nos. of Obs/Grand & $400 / 4260$ & $704 / 7480$ & $880 / 9350$ & $880 / 9350$ & $2864 / 30430$ \\
\hline Nos. of Instrument & 24 & 41 & 47 & 53 & 71 \\
\hline Nos. of Groups & 25 & 44 & 56 & 55 & 179 \\
\hline Wald test $\mathrm{p}$-value & 0.000 & 0.000 & 0.000 & 0.000 & 0.000 \\
\hline AR (1) $p$-value & 0.006 & 0.025 & 0.012 & 0.034 & 0.001 \\
\hline AR (2) $p$-value & 0.291 & 0.264 & 0.235 & 0.544 & 0.226 \\
\hline Hansen $p$-value & 0.583 & 0.311 & 0.341 & 0.272 & 0.315 \\
\hline
\end{tabular}

Source: Author's calculations

$\mathrm{t}$-statistics are in parentheses and all standard errors are two-step, significance: $* \mathrm{p}<0.1 ; * * \mathrm{p}<0.05 ; * * * \mathrm{p}<0.01$

Table 3 is the empirical results explaining the causal effects of OFDI on home country's imports as well as the spillover effects of other variables. OFDI from countries with Low income appears not to complement home country's imports of goods and services. In fact, OFDI flows for such countries can bring about $5.7 \%$ and $20.1 \%$ reduction in imports of goods and services. Table 3 estimation indicates that the effects of country's GDP for all income economies influences the imports of goods and services positively and significantly, Trade tariff impact imports negatively and significantly in low income economies. Negative trade tariff decreases flow of imported goods and services. This suggests that trade tariff in many poor countries are not favorable. Quality of infrastructure (QINF) in low and low-middle economies shows to negate inflow of imported goods and services. This could be due to poor infrastructure development such as poor telecommunication, bad road, poor railway, etc.

Table 4 shows the estimation of the reversed effects of OFDI on imports specified in model II in equation 2. The aim of this relation is to determine how the inflow of imported goods and services support OFDI flow. Results of our empirical analysis indicates that Imports of goods and services 
do not benefit OFDI flow in low income economies. The reason could be that the types of goods and services imported are not needed by MNCs. Unsolicited and poor quality of good and service usually finds its way to countries with low economies, where government are too poor or corrupt to have functional standard quality control department. Human capital (HDI) shows a significant effect on OFDI flow for countries in different income clusters (see Table 4). The robustness of our model specification shows adequacy as reported by the model diagnostics of Table $3 \& 4$, where both the Hansen tests of over identification and AR (2) autocorrelation tests proved insignificant. Table 5 reports the summary results for OFDI and international trade.

Table 3: Two-step system estimation results for effect of outward FDI on Imports

\begin{tabular}{|c|c|c|c|c|c|}
\hline & \multicolumn{4}{|c|}{ World Bank Income Classification } & \\
\hline & Low & Low-Middle & Upper-Middle & High & All Income \\
\hline Variables & (1) & $(2)$ & (3) & (4) & (5) \\
\hline Lagged IMP & $\begin{array}{l}0.782 * * * \\
(27.530)\end{array}$ & $\begin{array}{l}0.768 * * * \\
(84.910) \\
\end{array}$ & $\begin{array}{l}0.527 * * * \\
(96.460)\end{array}$ & $\begin{array}{l}0.704 * * * \\
(102.840)\end{array}$ & $\begin{array}{l}0.707 * * * \\
(79.160)\end{array}$ \\
\hline IFDI & $\begin{array}{l}0.077 * * * \\
(3.100)\end{array}$ & $\begin{array}{l}0.0312 \\
(0.204)\end{array}$ & $\begin{array}{l}0.516 * * * \\
(18.380)\end{array}$ & $\begin{array}{l}-0.029 * * \\
(-2.630)\end{array}$ & $\begin{array}{l}0.009 \\
(0.900)\end{array}$ \\
\hline OFDI & $\begin{array}{l}-0.057^{*} \\
(-1.830) \\
\end{array}$ & $\begin{array}{l}0.040 * * \\
(2.670) \\
\end{array}$ & $\begin{array}{l}0.037 * * * \\
(3.640)\end{array}$ & $\begin{array}{l}0.156 * * * \\
(-30.750) \\
\end{array}$ & $\begin{array}{l}0.039 * * \\
(2.100) \\
\end{array}$ \\
\hline GDP & $\begin{array}{l}0.715 * * * \\
(2.260) \\
\end{array}$ & $\begin{array}{l}0.426 * * * \\
(3.790)\end{array}$ & $\begin{array}{l}0.208 * * * \\
(3.340)\end{array}$ & $\begin{array}{l}0.617 * * * \\
(13.270)\end{array}$ & $\begin{array}{l}0.249 * * * \\
(3.830)\end{array}$ \\
\hline TEXP & $\begin{array}{l}-0.631 * * * \\
(-4.570)\end{array}$ & $\begin{array}{l}0.025 \\
(0.100)\end{array}$ & $\begin{array}{l}0.258 * * * \\
(15.820)\end{array}$ & $\begin{array}{l}-0.216 * * * \\
(-12.970)\end{array}$ & $\begin{array}{l}-0.652 * * * \\
(-4.100)\end{array}$ \\
\hline HDI & $\begin{array}{l}-0.302 \\
(-1.260)\end{array}$ & $\begin{array}{l}0.777 * * * \\
(4.190) \\
\end{array}$ & $\begin{array}{l}0.815 * * * \\
(7.440)\end{array}$ & $\begin{array}{l}-0.392 \\
(-1.370)\end{array}$ & $\begin{array}{l}-0.413 * * * \\
(-3.960)\end{array}$ \\
\hline TRDT & $\begin{array}{l}-0.186 * * \\
(-2.480)\end{array}$ & $\begin{array}{l}0.249 * * * \\
(4.620)\end{array}$ & $\begin{array}{l}0.440 * * * \\
(48.550)\end{array}$ & $\begin{array}{l}0.116^{* * * *} \\
(3.760)\end{array}$ & $\begin{array}{l}0.096 * * * \\
(2.670)\end{array}$ \\
\hline QINF & $\begin{array}{l}-0.087 \\
(-0.71) \\
\end{array}$ & $\begin{array}{l}-0.961 * * * \\
(-6.78)\end{array}$ & $\begin{array}{l}0.717 * * * \\
(8.380)\end{array}$ & $\begin{array}{l}0.326 * * * \\
(10.24)\end{array}$ & $\begin{array}{l}0.620 * * * \\
(6.410)\end{array}$ \\
\hline DEBT & $\begin{array}{l}-0.076 \\
(-0.710) \\
\end{array}$ & $\begin{array}{l}0.475 * * * \\
(4.280)\end{array}$ & $\begin{array}{l}0.241 * * * \\
(4.830)\end{array}$ & $\begin{array}{l}0.636 * * * \\
(12.440)\end{array}$ & $\begin{array}{l}0.035 \\
(0.680) \\
\end{array}$ \\
\hline Constant & $\begin{array}{l}1.798 * * * \\
(5.100) \\
\end{array}$ & $\begin{array}{l}1.978 * * * \\
(6.190) \\
\end{array}$ & $\begin{array}{l}-2.020 * * * \\
(-22.760)\end{array}$ & $\begin{array}{l}3.599 * * * \\
(13.580)\end{array}$ & $\begin{array}{l}1.735^{* * *} \\
(6.380)\end{array}$ \\
\hline Nos. of Obs/Grand & $400 / 4260$ & $704 / 7480$ & $880 / 9350$ & $880 / 9350$ & $2864 / 30430$ \\
\hline Nos. of Instrument & 22 & 42 & 48 & 52 & 83 \\
\hline Nos. of Groups & 25 & 44 & 56 & 55 & 179 \\
\hline Wald test $\mathrm{p}$-value & 0.000 & 0.000 & 0.000 & 0.000 & 0.000 \\
\hline AR (1) $p$-value & 0.006 & 0.005 & 0.012 & 0.034 & 0.000 \\
\hline AR (2) $p$-value & 0.217 & 0.264 & 0.199 & 0.631 & 0.275 \\
\hline Hansen $p$-value & 0.303 & 0.380 & 0.230 & 0.202 & 0.321 \\
\hline
\end{tabular}

Source: Author's calculations

$\mathrm{t}$-statistics are in parentheses and all standard errors are two-step, significance: $* \mathrm{p}<0.1 ; * * \mathrm{p}<0.05 ; * * * \mathrm{p}<0.01$ 
Table 4: Two-step system GMM estimation results for imports supporting outward FDI

\begin{tabular}{|c|c|c|c|c|c|}
\hline & \multicolumn{4}{|c|}{ World Bank Income Classification } & \multirow[b]{2}{*}{ All Income } \\
\hline & Low & Low-Middle & Upper-Middle & High & \\
\hline Variables & (1) & $(2)$ & (3) & (4) & (5) \\
\hline Lagged OFDI & $\begin{array}{l}0.222 * * * \\
(3.7400)\end{array}$ & $\begin{array}{l}0.148 * * * \\
(5.340)\end{array}$ & $\begin{array}{l}0.196 * * * \\
(8.110)\end{array}$ & $\begin{array}{l}0.139 * * * \\
(6.870)\end{array}$ & $\begin{array}{l}0.089 * * * \\
(3.420)\end{array}$ \\
\hline IFDI & $\begin{array}{l}0.120 * \\
(2.000) \\
\end{array}$ & $\begin{array}{l}0.105 \\
(1.430) \\
\end{array}$ & $\begin{array}{l}0.717 * * * \\
(14.680)\end{array}$ & $\begin{array}{l}0.362 * * * \\
(11.390)\end{array}$ & $\begin{array}{l}0.143 * \\
(1.720) \\
\end{array}$ \\
\hline IMP & $\begin{array}{l}-0.117^{*} \\
(-1.98) \\
\end{array}$ & $\begin{array}{l}0.072 * * * \\
(2.760)\end{array}$ & $\begin{array}{l}0.064 * * \\
(2.00)\end{array}$ & $\begin{array}{l}0.282 * * * \\
(-3.510)\end{array}$ & $\begin{array}{l}0.198 * * * \\
(5.170)\end{array}$ \\
\hline GDP & $\begin{array}{l}-0.340 * * \\
(-2.240)\end{array}$ & $\begin{array}{l}0.159 \\
(0.251) \\
\end{array}$ & $\begin{array}{l}0.717 * * * \\
(4.030)\end{array}$ & $\begin{array}{l}0.926 * * * \\
(13.870)\end{array}$ & $\begin{array}{l}0.817 * * * \\
(2.700)\end{array}$ \\
\hline TEXP & $\begin{array}{l}-0.842 * * \\
(-2.080)\end{array}$ & $\begin{array}{l}0.202 * * * \\
(2.900)\end{array}$ & $\begin{array}{l}0.613 * * \\
(2.140) \\
\end{array}$ & $\begin{array}{l}-0.135^{* * *} \\
(-17.140)\end{array}$ & $\begin{array}{l}-0.733 * * \\
(-2.600) \\
\end{array}$ \\
\hline HDI & $\begin{array}{l}0.049 \\
(0.760) \\
\end{array}$ & $\begin{array}{l}0.825 * * * \\
(3.100)\end{array}$ & $\begin{array}{l}0.376 \\
(1.300) \\
\end{array}$ & $\begin{array}{l}-0.299 * * * \\
(-5.40)\end{array}$ & $\begin{array}{l}0.872 * * * \\
(2.580)\end{array}$ \\
\hline TRDT & $\begin{array}{l}-0.432 * \\
(-1.800) \\
\end{array}$ & $\begin{array}{l}0.353 * * * \\
(5.400)\end{array}$ & $\begin{array}{l}-0.181 * * * \\
(-3.750) \\
\end{array}$ & $\begin{array}{l}0.206^{* *} \\
(2.580) \\
\end{array}$ & $\begin{array}{l}-0.204 * \\
(-1.710) \\
\end{array}$ \\
\hline QINF & $\begin{array}{l}0.238 \\
(0.450) \\
\end{array}$ & $\begin{array}{l}-0.310 * * * \\
(-4.220) \\
\end{array}$ & $\begin{array}{l}0.390 * \\
(1.710) \\
\end{array}$ & $\begin{array}{l}0.461 * * * \\
(6.230) \\
\end{array}$ & $\begin{array}{l}0.310 \\
(0.810) \\
\end{array}$ \\
\hline DEBT & $\begin{array}{l}-0.410 * * \\
(-2.280)\end{array}$ & $\begin{array}{l}-0.530^{*} \\
(-1.910) \\
\end{array}$ & $\begin{array}{l}-0.688 * * * \\
(-22.59)\end{array}$ & $\begin{array}{l}-0.857 \\
(-1.512) \\
\end{array}$ & $\begin{array}{l}-0.799 * * * \\
(-2.930)\end{array}$ \\
\hline Constant & $\begin{array}{l}1.619 * * \\
(2.070) \\
\end{array}$ & $\begin{array}{l}0.078 \\
(0.040) \\
\end{array}$ & $\begin{array}{l}-1.025^{* * *} \\
(-8.830) \\
\end{array}$ & $\begin{array}{l}5.217 * * * \\
(10.380)\end{array}$ & $\begin{array}{l}0.867 * \\
(1.890) \\
\end{array}$ \\
\hline Nos. of Obs/Grand & $400 / 4260$ & $704 / 7480$ & $880 / 9350$ & $880 / 9350$ & $2864 / 30430$ \\
\hline Nos. of Instrument & 24 & 39 & 47 & 48 & 92 \\
\hline Nos. of Groups & 25 & 44 & 56 & 55 & 179 \\
\hline Wald test $\mathrm{p}$-value & 0.000 & 0.000 & 0.000 & 0.000 & 0.000 \\
\hline AR (1) $p$-value & 0.006 & 0.005 & 0.012 & 0.034 & 0.000 \\
\hline AR (2) $p$-value & 0.291 & 0.234 & 0.211 & 0.714 & 0.115 \\
\hline Hansen $p$-value & 0.479 & 0.389 & 0.230 & 0.202 & 0.201 \\
\hline
\end{tabular}

Source: Author's calculations

$\mathrm{t}$-statistics are in parentheses and all standard errors are two-step, significance: $* \mathrm{p}<0.1 ; * * \mathrm{p}<0.05 ; * * * \mathrm{p}<0.01$

Table 5: Results summary of OFDI and International Trade

\begin{tabular}{|l|c|c|c|c|c|}
\hline Effects & \multicolumn{5}{|c|}{ Complementary (+) / Substitutionary (-) } \\
\hline Income classification & OFDI $\rightarrow$ EXP & EXP $\rightarrow$ OFDI & OFDI $\rightarrow$ IMP & IMP $\rightarrow$ OFDI \\
\hline Low Income & $(-)$ & $(-)$ & & $(-)$ & $(-)$ \\
\hline Low-middle Income & $(+)$ & $(+)$ & & $(+)$ & $(+)$ \\
\hline Upper-middle Income & $(+)$ & $(+)$ & & $(+)$ & $(+)$ \\
\hline High Income & $(+)$ & $(+)$ & & $(-)$ & $(+)$ \\
\hline All income & $(+)$ & $(+)$ & & $(+)$ & $(+)$ \\
\hline
\end{tabular}

\section{Conclusion}

This study has shed some light on the relationship between OFDI and international trade flows, with support for both theory of vertical and horizontal FDI. OFDI flows have a strong complementarity effects on exports in all income economies classification except countries with low income economies. Since countries characteristic differs in terms of relative endowments factors and low trade costs, the complementarity essence seeks to explore market integration. We found 
strong 'export-supporting' OFDI for most countries in different income groups. This implies that most countries' exports complement the OFDI flow. For linkages between outbound FDI and country's imports of goods and services, this paper document a positive bidirectional causation pattern for almost all countries' economies. However, OFDI appears to be a substitute for export in low-income countries. Therefore, policymakers from low income countries are expected to review, revise and implement FDI laws \& policies that attract and facilitate investments that complement sustainable economic developments.

\section{Acknowledgments}

This research is supported by the Russian President grant "Institutional determinants of foreign direct investment inflows: country and region level analysis" (grant No. MD 6402.2018.6)

\section{References}

Albulescu, C.T., \& Goyeau, D. (2019). The interaction between trade and FDI: The CEECs experience, International Economics and Economic Policy, 16(3), pp. 489-509

Alguacil M, Cuadros A, \& Orts V (2008). EU enlargement and inward FDI. Review of Development Economics 12, 594-60.

Aziz, O.G. (2018). Institutional quality and FDI inflows in Arab economies, Finance Research Letters, Volume 25, Pages 111-123, https://doi.org/10.1016/j.frl.2017.10.026.

Andrenelli, A., Cadestin, C., De Backer, K., Miroudot, S., Rigo, D., \& Ye, M., (2018). Multinational production and trade in services, OECD Trade Policy Papers 212, OECD Publishing, https://dx.doi.org/10.1787/16ec6b55-en

Blundell, R. \& Bond, S. (1998). Initial Conditions and Moment Restrictions in Dynamic Panel Data Models, Journal of Econometrics, 87, pp. 115-143.

Bhasin, N. \& Kapoor, K. (2020). Impact of outward FDI on home country exports, International Journal of Emerging Markets, Vol. ahead-of-print No. ahead-of-print, https://doi.org/10.1108/IJOEM-05 2017-0160

Dauti, B. (2016). Trade and foreign direct investment: Evidence from South East European countries and new European Union member states, Proceedings of Rijeka School of Economics 34(1):63-89 DOI:10.18045/zbefri.2016.1.63

Joshua, U., Rotimi, M. E., \& Sarkodie, S. A., (2020). Global FDI Inflow and Its Implication across Economic Income Groups, Journal of Risk Financial Management, 13, no. 11: 291. https://doi.org/10.3390/jrfm13110291

Krautheim, S. (2013). Export-Supporting FDI. Canadian Journal of Economics/Revue Canadienne d'e'conomique 46(4),1571-605. http://dx.doi.org/10.1111/caje.12061.

Zhi-yuan, L. (2017). Inward FDI Influences Posed on Chinese Exports, 4th International Conference on Economics and Management (ICEM), Pages, 208-212, 10.12783/dtem/icem2017/13109 
Lorz, O. (2020). Investment in trade facilitating infrastructure: A political-economy analysis, European Journal of Political Economy, Volume 65, 101928, https://doi.org/10.1016/j.ejpoleco.2020.101928.

Martínez-San Román, V., Bengoa, M. \& Sánchez-Robles, B. (2016). Foreign direct investment, trade integration and the home bias: evidence from the European Union. Empirical Economics 50, 197 229. https://doi.org/10.1007/s00181-015-0942-y

Mazouz, K., Wood, G., Yin, S., \& Zhang, M. (2021). Comprehending the outward FDI from Latin America and OCED: A comparative perspective, International Business Review, 101853, https://doi.org/10.1016/j.ibusrev.2021.101853.

Sharmiladevi, J. C. (2020). Cointegration and Causality Study Among Inward FDI, Economic Growth and Exports: An Indian Perspective. International Journal of Asian Business and Information Management (IJABIM), 11(1), 63-77. http://doi.org/10.4018/IJABIM.202001010

Ismail, N.W. (2021). Digital trade facilitation and bilateral trade in selected Asian countries, Studies in Economics and Finance, Vol. 38 No. 2, pp. 257-271. https://doi.org/10.1108/SEF-10-2019-0406

UNCTAD, (2021). Global foreign direct investment falls 49\% in first half of 2020, Palais des Nations, 8 14, Av. de la Paix, 1211 Geneva 10, Switzerland

UNCTAD, (2012). Global FDI Outflows Continued to Rise In 2011 Despite Economic Uncertainties; However, Prospects Remain Guarded, Global Investment Trend Monitor,No: 9, New York, NY: United Nations.

WTO, (2019), World Trade Statistical Review, Geneva, Switzerland

Zhou, C. (2020). The effects of outward FDI and export on firm productivity in emerging markets: Evidence from matching approach, Economics Letters 195,109462. https://doi.org/10.1016/j.econlet.2020.109462

Zhao, L., Liu, Z., Wei, W., \& Andreosso-O'Callaghan, B. (2017). FDI outflows, exports and financial development, Journal of Economic Studies, 44(6), pp. 987-1002 